\title{
Age Relevant Dentistry for the Mature Patient
}

\section{Fred C Quarnstrom*}

American Dental Board of Anesthesiology, American Association of Dental Consultants, University of Washington, Seattle, USA

Dental needs of elderly patients often differ significantly from those of young patients. Elderly patients need to deal with the "ravages of time", disease, unhealthy life style, bad habits, poor diet, genetics, lack of dental treatment and excessive or unwise dental treatment. As dentists, we are trained to treat the young differently than adults. Little thought is given to the potential life span of the patients when prescribing dental treatment once a patient is past the age of ten.

Periodontal disease has a very strong genetic component. In general, it progresses with age. The rate of breakdown seems primarily dictated by genes that affect the inflammatory process. Progression can be slowed down by careful home care and professional treatment. Sometimes, the best a dentist can hope to accomplish is to slow the progression of bone loss. If the periodontal disease is refractory to treatment, it will progress; and, the patient's dental future will be very different than those without the defective genes. Contrary to what many dental websites state, taking care of one's periodontal disease will not prevent heart attacks, strokes or low birth weight babies. All are affected by the compromised inflammatory system. Research shows correlation, not causality, two very different things.

The patient needs to consider what dentistry they need and what results they want to see. Do they need the treatment to last 10,20 , or 30 years? If one is in their 40 's or 50 's, the answer is clearly that a longer lasting treatment is more appropriate (Figure 1).

If one is $80+$, the techniques and solutions that the dentist provides will need to be different. Should the dentist alter the treatment, using materials that can be repaired? should there be future decay (Figure 2)? If the patient gets a gold crown, it can be repaired with a silver filling. If, instead, they get an all porcelain crown, it will look great, but it cannot be repaired because no material will bond to porcelain. If the patient is 60 years old and has a gold crown that gets decayed at 85 , the crown can be repaired with a simple silver filling that may last 15 years or more (Figure 3). But if they choose a porcelain crown and decay occurs, they will need another crown. A repair will be more cost effective and less stressful for the patient. At the age of 85 , particularly if the patient has some medical problems, this decision can be life saving. Repairs are

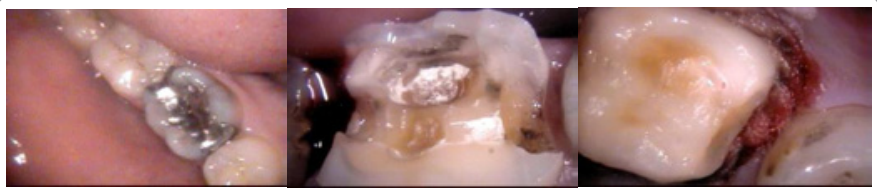

Figure 1: An MOD alloy was removed for an all porcelain crown in 27 years old patient is in the process where the pulp was exposed.

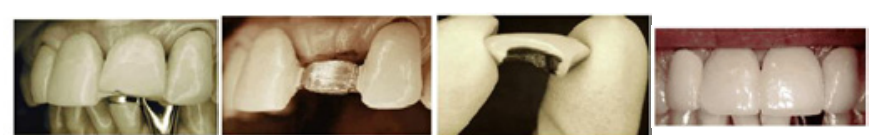

Figure 2: A 70 years old patient on a fixed income with no insurance fractured the left central on a 4 tooth bridge, $6-10$. The porcelain was removed and the metal conector preped. A pontic was constructed to draw to the Labial. The final result (right image) is acceptable.

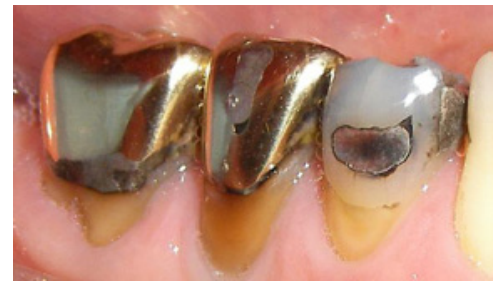

Figure 3: The patient is 74 years old Crowns were done 30 years earlier. The repair on the molar was done 15 year later. The bicuspids need repairs.

often the best solution. They cause less trauma to the tooth and nerve and extend the life of the filling or crown for years, at a fraction of the cost of a new crown.

The questions that should have been considered are:

1. How will no treatment effect the patient?

2. What treatment options exist?

3. What does the patient desire?

4. How will the treatment effect the patient's future health?

5. How will the treatment effect the tooth?

6. Which treatment options can be repairable in the future?

7. What is the financial impact to the patient of each option?

8. Does the patient and/or their family understand all the considerations?

Aesthetic dentistry, smile design, perfectly white teeth are the watchwords of the $21^{\text {st }}$ century. If one's teeth are not the color of new fallen snow, they are substandard. For this reason many people choose all porcelain crowns. If secondary decay occurs, the porcelain crowns will need to be redone. If the patient got the perfect smile makeover at 60 to make them look 50, while he had dental insurance, will they have the financial resources to redo these, at 80 , if the beautiful crowns begin to fail? If the makeover cost $\$ 28,000$ in 2000 , it will probably cost $\$ 40,000$ in 2010 and $\$ 70,000$ in 2020 . The redo may not be possible if the patient is on a fixed income. Will there be enough tooth structure left to allow a redo? Will they be healthy enough to contend with hours in the dental chair when the components of that perfect smile start

*Corresponding author: Fred C Quarnstrom, D.D.S, Dental Anesthesiologist American Dental Board of Anesthesiology, American Association of Dental Consultants, University of Washington, Seattle, USA, Tel: (206) 329-0500; Fax (206) 329-0538; E-mail: quarn@uw.edu

Received March 28, 2012; Accepted August 03, 2012; Published August 05 2012

Citation: Quarnstrom FC (2012) Age Relevant Dentistry for the Mature Patient Dentistry S3:001. doi:10.4172/2161-1122.S3-001

Copyright: @ 2012 Quarnstrom FC. This is an open-access article distributed under the terms of the Creative Commons Attribution License, which permits unrestricted use, distribution, and reproduction in any medium, provided the original author and source are credited. 


\section{Professionally Applied Topical Fluoride: Evidence-based Clinical Recommendations ${ }^{1}$}

\section{Determination of Caries Risk}

There are many systems to determine caries risk. One such system is offered below that can be used for caries risk assessment.

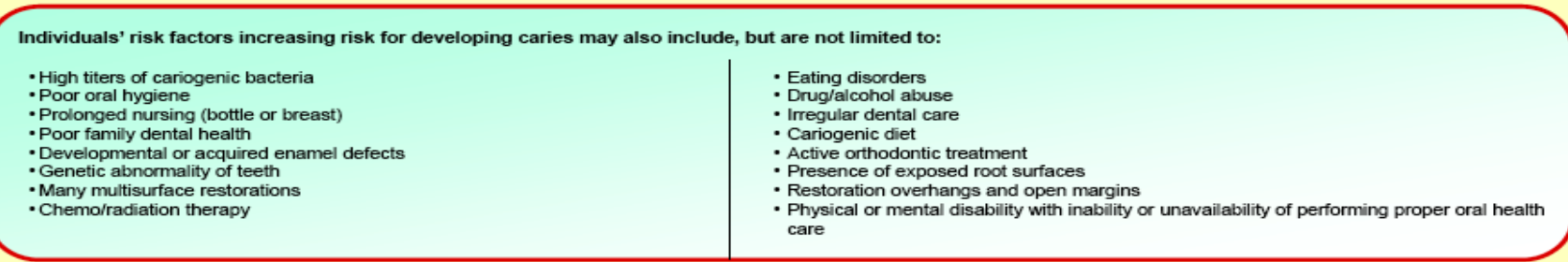

\begin{tabular}{c|c|c|c|c|}
\hline \multirow{2}{*}{ Risk group } & Age & Primary or Secondary Carious lesions in the past & & Risk factors listed above \\
\hline Low & All age groups & None & and & None \\
\hline \multirow{2}{*}{ Moderate } & $<6$ years & None & and & At least one risk factor \\
\cline { 2 - 5 } & $>6$ years & One or two & or & At least one risk factor \\
\hline \multirow{2}{*}{ High } & $<6$ years & Any & $\begin{array}{c}\text { Multiple risk factors or Low Socioeconomic status or } \\
\text { Xerostomia* or suboptimal fluoride exposure }\end{array}$ \\
\cline { 2 - 5 } & $>6$ years & Three or more & or & $\begin{array}{c}\text { Multiple risk factors or Xerostomia* or suboptimal } \\
\text { fluoride exposure }\end{array}$ \\
\cline { 2 - 6 } & & &
\end{tabular}

${ }^{*}$ Medication, radiation or disease induced xerostomia.

\section{ADA Council on Scientific Affairs. Professionally applied topical fluoride: Evidence-based clinical recommendations. JADA 2006;137(8):1151-59. Copyright @ 2006 American Dental Association. All rights reserved. Adapted 2008 with permission. To see the full text of this article, please go to http://jada.ada.orq/cqi/reprint/137/8/1151.}

Thls page may be used. copled. and distributed for non-commercial purposes without obtaining prior approval from the ADA. Any other use, oopying, or distribution, whether in printed or electronic format, is stricty pronibibed without
the prof wr wen consent of the ADA.

Figure 4: Factors that complicate the issue include the need for sinus lifts and additional surgery to do implants.

breaking down. That patient might be better off without the miracle makeover (Figure 4).

Americans are living longer and longer; but, it is not possible to predict life span. I think of three patients:

1. Patient $\mathrm{A}$ is 55 years old, $70 \mathrm{lbs}$ overweight, probably has sleep apnea and has had two heart attacks. Clearly, this patient has health problems. He has good insurance and many dental needs. We need to consider whether a dental procedure is really needed, and whether he can handle the stress of the proposed treatment. His insurance will pay, but does he really need extensive dentistry?

2. Patient $B$ is 93 years old and exercises on a rowing machine 45 minutes each night. He has had a pacemaker for 20 years but is alert, drives his car, and lives alone. We again worry a little about the stress of dental procedures and probably do not have to perform dentistry that will last 20 years. He is wealthy and can and will accept any treatment we suggest.

3. Patient $\mathrm{C}$ is on a limited income, social security, has severe wear of all his teeth. His upper front teeth have been worn thin. $\mathrm{He}$ is 93 years old and hand cultivates a garden that is over one half acre. He takes no medicine and has no serious medical problems in his past.

Should we treat all three patients the same? The best treatment is very different for these three (Figure 5).

Extensive dentistry can only be considered while the patient is healthy enough to stand the stress of providing the treatment. With the passage of time, they will be less robust. The patient's age and degenerative disease states may make the stress of medication and long dental appointments unacceptable. While any dentist would like to think that what he does will last a lifetime, many factors come into play. As patients get older, their hand-eye coordination decreases making it harder to brush, floss and properly clean their teeth. Those with dementia, Alzheimer's disease, not only are less capable, they need help to remember to care for their mouths. Various medical conditions and medications can lead to loss of salivary flow. Many things can cause a patient who was perfect at homecare, to digress to a level of cleaning that leads to rapid breakdown. Teeth become more prone to fracture.

Patients with certain conditions can experience breakdown that we would never have expected. In such situations, it is kinder to repair existing fillings. Due to advanced age and/or medical complications, these patients may not be able to tolerate starting over. The economics of redoing major dental restorations may be impossible for those on a fixed income. The patient may rightly say it does not make sense to do that "at my age". Hopefully his dentist will select procedures that can be repaired in such cases. These repairs should be quick, low stress and, ideally, inexpensive. Fortunately, the pulp chambers of these patients have often receded to the point that these repairs can be done without local anesthesia because they cause no pain. Local anesthesia is remarkably safe, but still is a major stress producer in many dental procedures.

From a practical perspective based on my forty-six years in the practice of dentistry, I am convinced that some rather simple repairs are the best solution to many problems we see in the elderly. There is some science to show this is the correct approach. Alloy fillings can 


\section{Professionally Applied Topical Fluoride: Evidence-based Clinical Recommendations ${ }^{1}$}

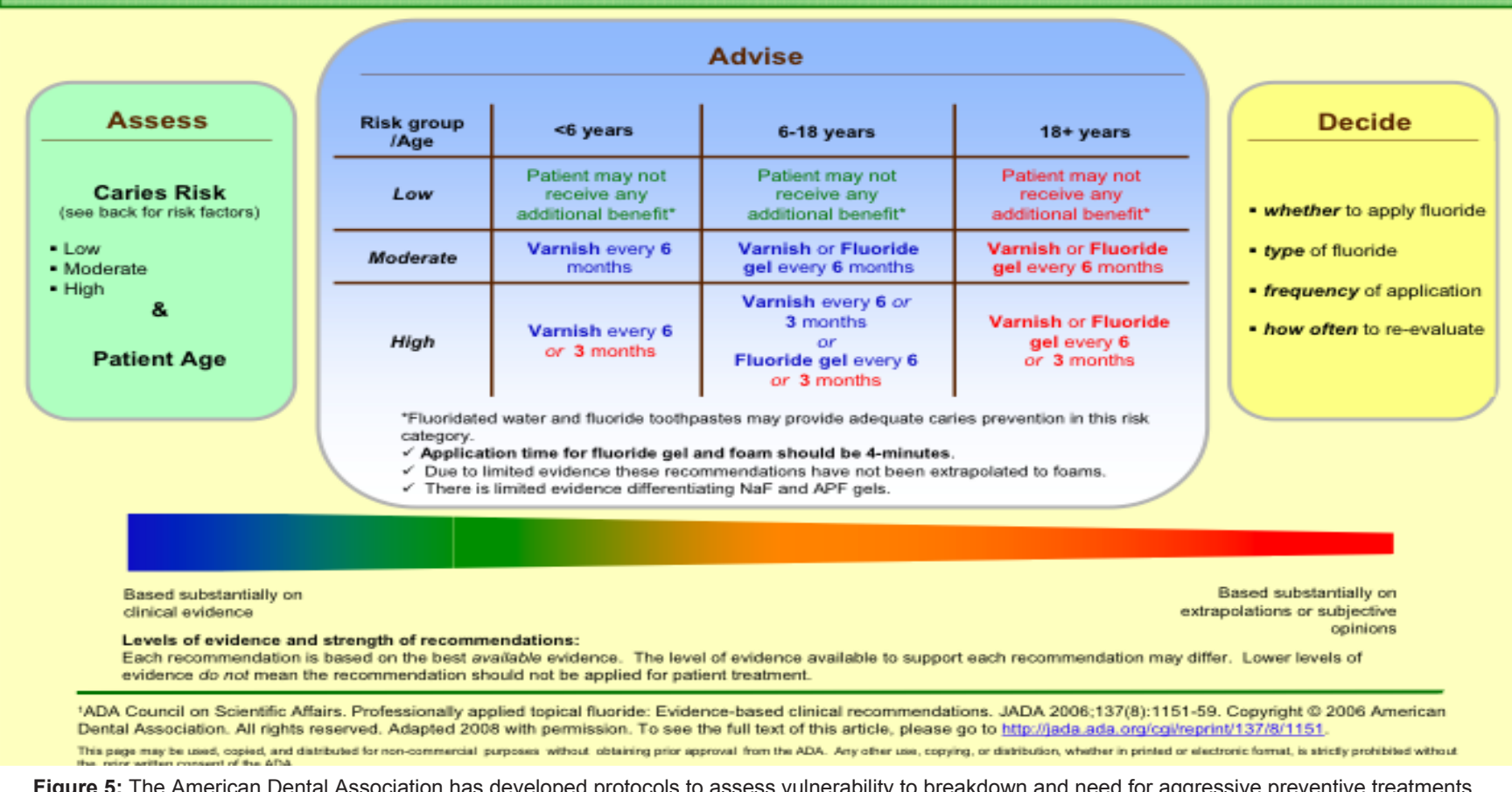

be repaired with small alloys to stop decay. The repairs replace small defects when tooth structure is lost due to chipping or small areas of decay. In fact, repairs may be a better solution than replacing the whole restoration or going to a cast restoration. Bonding to a composite may be less predictable. Alloy repairs can be applied to cast restorations, gold inlays and crowns. We have shown that it is possible to repair pontics and the abutments where porcelain has broken, without replacing the whole bridge.

Consider a patient who is 90 years old, had a heart attack 10 years earlier but survived with coronary vessel dilatation. In all other ways he is quite healthy. He had many of his teeth crowned 30 and 40 years ago. In some of these, the pulp died and the patient needed root canal therapy. In time, some of the crowns broke off with much of the coronal portion of the tooth in the crowns. Many of these teeth had posts and cores to preserve the crowns. Some of the teeth fractured down the root and were lost. In his upper arch, he had three sound teeth. He is wearing a partial denture, but he is not happy with it because it is not stable. He had considered implants 10 years ago but his insurance did not cover implants at that time. He does have some coverage of implants now. His options include:

1. Extractions and a full upper denture - cost about $\$ 3,000$; but at best loose and a compromise when chewing. Adapting to a full denture will be problematic.

2. Placing 4-implants and using a hybrid denture - cost about $\$ 14,000$, tighter but hard to clean around and under, but it can be repaired if it fractures.

3. Placing 6 implants and a fixed bridge - cost about $\$ 35,000$, as close as he can get to having teeth again but not possible to repair if it fractures.
4. Doing nothing - cost nothing but is not as stable as option \#1.

The issue that led us to this patient's present quandary is the result of crowning so many teeth in his 40s and 50s. Many of the crowns had to be redone over the years, leaving less tooth structure. Could these have been repaired rather than being recrowned? Were the crowns done for functional reasons, or because a cast restoration looked better and there was good insurance that would cover crowns? It is too late for these questions for this patient. As a patient ages, there are good reasons to do whatever can be done to protect the margins of the crowns. The margin where the tooth meets the root is an area that is very vulnerable to future decay.

Children routinely receive fluoride treatments. Fluoride varnish and silver fluoride are two of the most successful. Fluoride varnish as its name implies is fluoride mixed with varnish. The varnish is painted on the teeth, tooth roots, and crowns. It is set with a water rinse. Once on the teeth, it continues to release fluoride ions for 10 to 20 weeks. It is particularly effective in the groves and any defects where crowns meet the tooth surfaces particularly the roots. A second approach is to have the patients brush with a $1 \%$ fluoride gel each night before going to sleep. Protocols have been developed to remove stress with the use of fluoride varnish on a regular basis in all patients at risk of decay by antibacterial rinses, peridex, and xylitol gums and mints (Figure 6).

Other options including the use of Silver fluoride has been well researched in the Scandinavian countries along with China and Japan. This clear liquid can be applied to an area of decay. It stains the decay dark black, stops the decay and prevents future decay. It must be carefully applied, as it will also stain the gingival tissue and lips black if it touches these areas. 

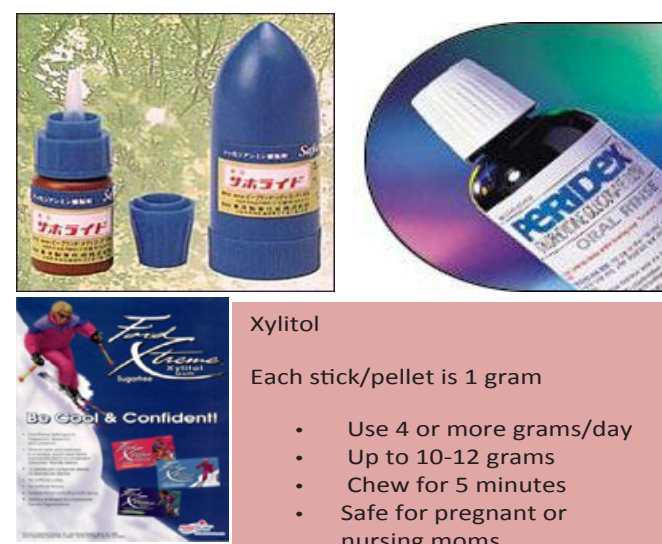

\section{Xylitol}

Each stick/pellet is $1 \mathrm{gram}$

Use 4 or more grams/day

Up to 10-12 grams

Chew for 5 minutes

Safe for pregnant or nursing moms

Figure 6: Protocols to remove stress with use of fluoride varnish.

As our population ages, more and more patients who selected procedures that were offered and performed at $30,40,60$, or 70 years of age, now present us with problems that are nearly impossible to solve. We need to do a better job of planning treatment to allow for the issues our patients will face as they age. Just because we can perform a procedure today, does not necessarily mean that that particular choice will be the best for the future.

Dental insurance is not available to most patients once they retire. As they transition to a decreased fixed income they find all dental costs coming out of their pockets. Dental coverage is not part of Medicare or Medicare supplement programs and dental insurance is lost when they retire. At the time when these patients are most vulnerable to breakdown, the patients are on a decreased fixed income; and have no access to dental insurance. Some of their woes are the result of insurance companies that auto adjudicate rather than reviewing requests for treatment that are inappropriate. The inappropriate treatments rendered the teeth vulnerable to breakdown at this vulnerable time.

Age relevant treatment should become an important part of the dentist and their patient's decision making. We simply have to stop treating for today and consider the consequences our treatments will have for the patient as they age. Third party plans must revaluate there positions to be sure that their policies do not further this sacrifice of dentine and enamel. 